\title{
Upfront debulking surgery for high-grade serous ovarian carcinoma: current evidence
}

\author{
Orestis Tsonis ${ }^{1}$, Fani Gkrozou ${ }^{2}$, Konstantinos Vlachos ${ }^{3}$, Minas Paschopoulos ${ }^{1}$, Michail C. Mitsis ${ }^{3}$, \\ Nikolaos Zakynthinakis-Kyriakou ${ }^{4}$, Stergios Boussios ${ }^{5,6}$, George Pappas-Gogos ${ }^{3}$ \\ ${ }^{1}$ Department of Obstetrics and Gynaecology, University Hospital of Ioannina, Ioannina, Greece; ${ }^{2}$ Department of Obstetrics and Gynaecology, \\ University Hospitals Birmingham, Birmingham, UK; ${ }^{3}$ Department of General Surgery, University Hospital of Ioannina, Ioannina, Greece; ${ }^{4}$ Leicester \\ Diabetes Research Centre, Leicester, UK; ${ }^{5}$ Department of Medical Oncology, Medway NHS Foundation Trust, Gillingham, Kent, UK; ${ }^{6}$ AELIA \\ Organization, Thessaloniki, Greece \\ Contributions: (I) Conception and design: G Pappas-Gogos, S Boussios; (II) Administrative support: None; (III) Provision of study materials or \\ patients: None; (IV) Collection and assembly of data: O Tsonis, F Gkrozou; (V) Data analysis and interpretation: G Pappas-Gogos, S Boussios; (VI) \\ Manuscript writing: All authors; (VII) Final approval of manuscript: All authors. \\ Correspondence to: Dr. Stergios Boussios, MD, PhD, MRCP (London). Consultant Medical Oncologist, Medway NHS Foundation Trust, Windmill \\ Road, ME7 5NY, Gillingham, Kent, UK. Email: stergiosboussios@gmail.com; stergios.boussios@nhs.net.
}

\begin{abstract}
High-grade serous ovarian carcinoma (HGSOC) is a leading cause of mortality among women worldwide. Currently, there is no clear consensus over the regime these patients should receive. The main two options are upfront debulking surgery with adjuvant chemotherapy or neoadjuvant chemotherapy followed by interval debulking surgery (IDS). The former approach is proposed to be accompanied by lower chemoresistance rates but could lead to severe surgical comorbidities and lower quality of life (QoL). Optimizing patient's selection for upfront debulking surgery might offer higher progression-free and overall survival rates. Further studies need to be conducted in order to elucidate the predictive factors, which are favorable for patients undergoing upfront debulking surgery in cases of high-grade serous ovarian cancer.
\end{abstract}

Keywords: Upfront debulking surgery; high-grade serous ovarian cancer; interval debulking surgery (IDS); chemotherapy; cytoreductive surgery

Submitted Feb 15, 2020. Accepted for publication Apr 17, 2020.

doi: $10.21037 / \mathrm{atm}-20-1620$

View this article at: http://dx.doi.org/10.21037/atm-20-1620

\section{Introduction}

Ovarian cancer remains one of the main causes of death for women worldwide and although novel scientific evidence on its origin, genetics, prognosis and treatment has been accumulated over the years, low survival rates are consistent $(1,2)$.

High-grade serous ovarian carcinoma (HGSOC) is a subtype mainly responsible for deaths due to ovarian cancer, rather than all the other subtypes combined $(1,3)$.

Traditionally, cytoreductive surgery prior or after platinum-based chemotherapy comprise the gold standard of therapy for HGSOC $(3,4)$. Nevertheless, surgical treatment options have long been debated and whether upfront debulking in cases of HGSOC or neoadjuvant chemotherapy followed by interval debulking surgery (IDS) are appropriate, remains still controversial. In this review, current evidence on upfront debulking surgery for high-grade ovarian carcinoma is presented (5). Better understanding on patient's selection, prognostic factors and advantages of this approach will facilitate surgeons' decision on optimal timing for cytoreductive surgery.

\section{Prognostic factors for upfront debulking surgery}

Upfront debulking surgery necessitates a strict selection of patients based on specific prognostic factors and could potentially indicate the group of patients suffering from HGSOC, which will benefit the most by undergoing 
upfront cytoreduction (6). Usually, if cytoreduction to no residual disease is feasible, upfront debulking surgery is preferable rather than neo-adjuvant chemotherapy followed by IDS (3). Biomarkers, histologic and genomic factors, intestinal involvement, extra-pelvic disease, hormone levels and patient's profile are just few of the proposed prognostic factors that should be taken into consideration prior to surgical decision $(7,8)$.

\section{Predictive biomarkers for response to (neoadjuvant) chemotherapy}

Several biomarkers for optimal response to neoadjuvant chemotherapy have been proposed, although current evidence remain controversial regarding their potential use. Mesothelin, FLT4, $\alpha-1$ acid glycoprotein (AGP) and cancer antigen 125 (Ca-125) are proposed as suggestive of antivascular epithelial growth factor (anti-VEGF) monoclonal antibody success as a first line standard chemotherapy $(9,10)$.

Angiogenesis and vascular remodeling are complex processes that involve regulation by the cytokines angiopoietin-1 (Ang1) and Ang2, which interact with the vascular receptor tyrosine kinase Tie2. However, further studies are required in order to be clarified their predictive value (10).

\section{Histologic and genomic factors}

In contrast to all the other subtypes, high-grade serous ovarian cancer may be ideal for neoadjuvant chemotherapy, since this subtype is accompanied by higher chemosensitivity $(3,10)$. Genomic factors, such as cyclin E1 amplifications and the presence of breast cancer BRCA1/2 mutations, have also been suggested as predictive for optimal surgical timing, by defining chemo-resistant over chemo-sensitive high grade-serous ovarian cancer patients $(3,11)$. It has been estimated that approximately $1.3 \%$ of women will eventually develop ovarian cancer during their lives $(12,13)$. In this group, it has been documented that approximately $44 \%$ of women who inherit BRCA1 mutation and approximately $17 \%$ of women who inherit BRCA2 mutation will develop ovarian cancer by their eighties (14-16). BRCA mutation, especially BRCA2 patients, had higher response rates in neoadjuvant therapies and could benefit from this regime $(15,17)$. Patients with BRCA mutation have shown greater rates of overall survival, longer disease-free interval (DFI) after first-line chemotherapy, better responsiveness in common chemotherapeutic regimens and higher treatment free interval (TFI) between each line of therapy $(18,19)$. For women with BRCA1/2 mutations, progression-free survival is estimated at 15.7 and 21.6 months respectively, and overall survival for both groups were approximately 55.3 and 75.2 months, respectively $(15,20)$. In patients with ovarian carcinomas showing no BRCA 1 or 2 mutation, progression-free survival and overall survival were estimated at 16 and 56 months, showing great similarities to BRCA1 rates $(21,22)$.

\section{Tumor-infiltrating lymphocytes (TILs), tumor cell-free $D N A(c f D N A)$}

TILs and cfDNA have also been suggested as promising predictive biomarkers although their use is limited and no standard methods for their isolation have been suggested $(10,23)$.

More specifically, high levels of TILs were associated with better response to neoadjuvant chemotherapy, showing that host immune response influences the tumour chemosensitivity (24-26). Additionally, cfDNA levels rises in cases of advanced ovarian cancer. Indeed, high levels of these short fragments of nucleic acids are related to poor overall survival rates and might serve as adequate independent prognostic factor of neoadjuvant chemotherapy. Their value for the screening process is superior to $\mathrm{Ca}-125$, including both sensitivity and specificity $(24,27)$.

\section{Age, performance status, comorbidities, albumin, and body mass index}

Patient's status was a good prognostic factor with regards to patient's selection for upfront debulking surgery. A scoring system evaluating Body Mass Index of the patient, Ca-125 levels and imaging staging was conducted, and a group of scientists tried to identify patients who would gain benefit from primary cytoreductive surgery. Patients with BMI $<30 \mathrm{~kg} / \mathrm{m}^{2}, \mathrm{Ca}-125<100 \mathrm{IU} / \mathrm{L}$ and absence of PET/CT findings suggestive of either diaphragmatic and omental carcinomatosis, or positive parenchymal metastases were chosen to undergo upfront debulking surgery with lower risks of no complete cytoreduction (28). In general age and general performance status of the patient will eventually affect the treatment for cases of high-grade serous ovarian cancer.

Patients older than 65 years of age, with albumin levels $<25 \mathrm{~g} / \mathrm{L}$ and ascites $>1 \mathrm{~L}$ were less likely to benefit from upfront debulking surgery. 


\section{Intestinal resections}

Although cytoreductive surgeries in patients with advancedstage ovarian cancer are considered the most challenging in gynecologic oncology, Griffiths has showed that there is survival benefit if the residual tumor after debulking surgery does not exceed $15 \mathrm{~mm}$ in diameter (29). Despite the fact that surgical interventions aim to the total resection of the tumor, ensuring zero macroscopic residual tumor or complete (R0) cytoreduction, macroscopic tumor residual less than $10 \mathrm{~mm}$ is considered optimal (30).

The strategy of multiorgan resections represents a common practice. Most often, parts of the digestive tract should be resected, especially the colon and/or the rectum. The ortho-sigmoid resection in patients with bulky pelvic disease is rational, because distal sigmoid is often involved either by direct expansion or by implantation of ovarian cancer in the serosa, and this surgical excision can be performed with acceptable peri-operative morbidity. The most severe complication in relation to intestinal surgery is anastomotic dehiscence, which occur especially in rectosigmoid resection with low anastomosis. The incidence of this complication is $2.8-23 \%$ for colonic cancer resections and $0.8-6.8 \%$ for gynecologic cancers (31-34). Protracted surgery, blood transfusion, and short distal segment of anastomosis represent the factors most implicated in the risk for anastomotic dehiscence. Some authors suggest the performing of diverting ileostomy or colostomy in order to avoid or minimize such complication.

Others reported that the overall rate of complications associated with en-bloc resection of the uterus and adnexa with recto-sigmoid without protective ileostomy is around $2 \%$.

Therefore, the stomas should be performed only in situations such as poor nutrition, significant ascites, anastomosis under tension or multiple anastomoses. Regarding Hartmann's procedure, is considered appropriate for patients with significant comorbidities (35-37).

Several studies have focused on the impact of extended surgery on survival. Scarabelli et al. reported $100 \%$ 2 -year survival for patients without macroscopic tumor after surgery and $77 \%$ for patients with implants of $1 \mathrm{~cm}$ diameter. Patients with residual disease $>2 \mathrm{~cm}$ had not reached 2 years (38). Another study from Takahashi and coauthors showed $60.8 \%$ of 5 -year cumulative survival in patients without residual disease, while patients with macroscopic disease had 0\% (39). Cumulative 5 -year survival in patients undergoing primary radical cytoreductive surgery with bowel resection was $62.2 \%$, while in patients operated after neoadjuvant chemotherapy, survival was only $13.9 \%$ (38). A study from Arora reported 2 -year disease-free survival in $63 \%$ of patients who achieved optimal cytoreduction with bowel resection (40).

Peiretti et al. showed 72 months median overall survival time among patients with complete cytoreduction compared with 42 months among the rest (41). Estes and colleagues have showed improved disease-free survival in patients with advanced epithelial ovarian carcinoma undergo bowel resection as part of optimal cytoreduction, combined with platinum and taxane, as well as a tendency to improve overall survival (42).

Bowel resection allows for optimal cytoreduction in patients with advanced ovarian cancer and thus improves overall survival. Postoperative morbidity and mortality can be minimized by careful patient selection, techniques such as stomas creation and close postoperative observation. Intestinal surgery represents a decisive part of the debulking procedures for optimal cytoreduction. In patients with advanced disease who are expected to have optimal residual tumor, resection of more than two bowel parts can be feasible and safe with acceptable rates of complication. Subtotal colectomy should be considered in selective patients due to high rates of anastomotic leakage (43).

\section{Peritoneal carcinomatosis}

Obviously, strong indicator favouring neoadjuvant chemotherapy over upfront debulking surgery is the possibility of tumour resection. In other words, unresectable disease or extent generalised carcinomatosis are current indications for neoadjuvant chemotherapy (24).

Moreover, spread of the disease appears to be decisive for abandoning upfront debulking surgery. Deep infiltration or diffuse metastasis within both small and large bowel could lead to severe surgical morbidities (10). Celiac lymph node involvement is accompanied with higher rates of large bowel resection and higher incidence of metastasis to small bowel mesentery (44). Lymph node involvement in general does not seem to favorite primary cytoreductive surgery, while peritoneal carcinomatosis increases the rates of cytoreductive failure and surgical complications, if upfront debulking surgery is decided $(10,45,46)$.

\section{Extrapelvic disease (Fagotti scoring system and ascites)}

Laparoscopic index of Fagotti is a 100-point score relevant in prediction of optimal cytoreduction among women undergoing IDS. Predictive parameters include peritoneal 


\section{Page 4 of 11}

Table 1 Prognostic factors favoring upfront debulking surgery in patients with high-grade serous ovarian cancer

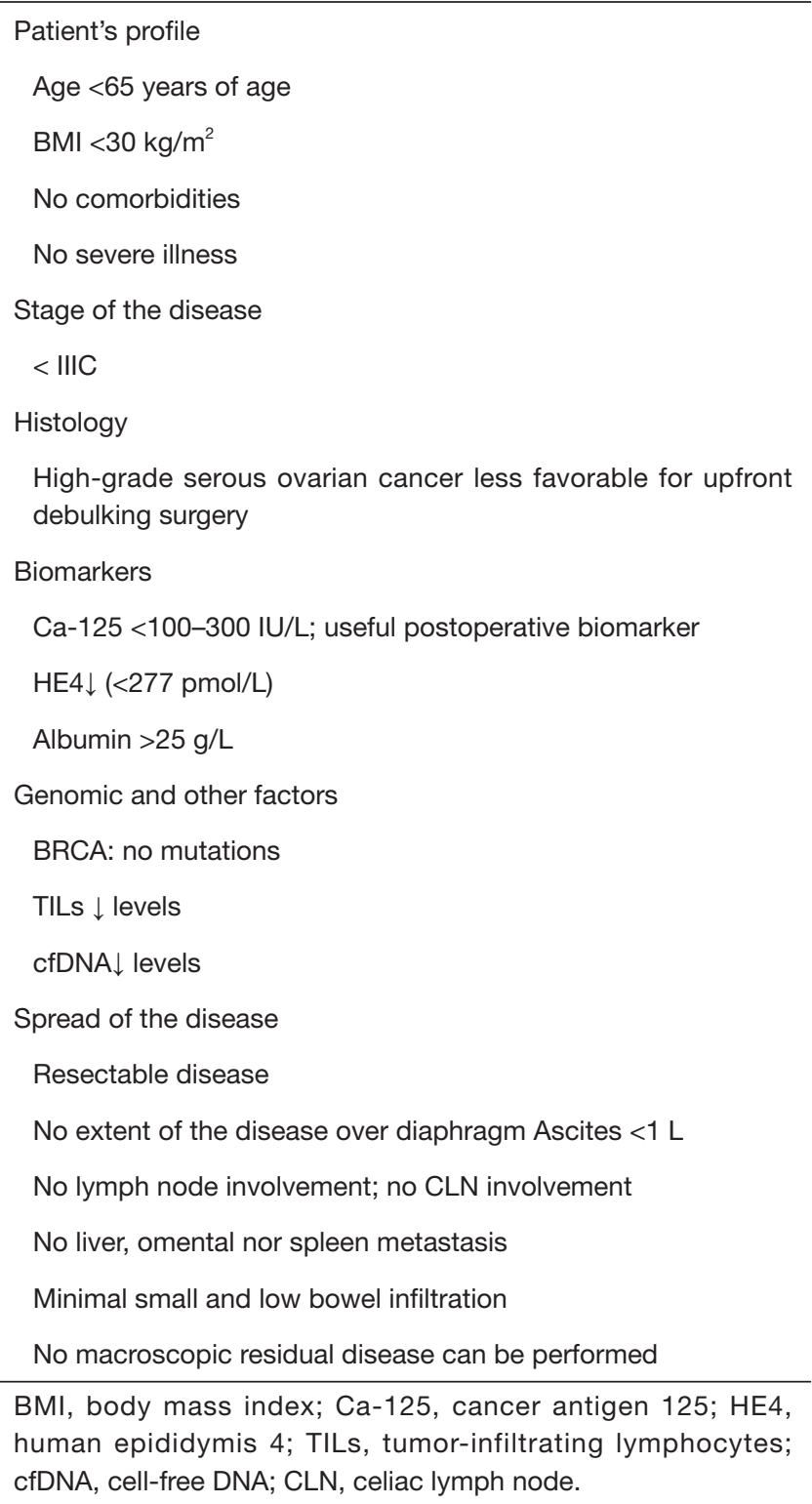

carcinomatosis, diaphragmatic and mesenteric disease, omental metastasis, bowel and stomach infiltration and liver metastases, which underline the influence of extraperitoneal and metastatic disease in surgical prognosis $(18,47)$. Patients are classified into three risk groups of incomplete cytoreduction. Those at high-risk would be treated with neoadjuvant chemotherapy, whereas low-risk patients may benefit from upfront complete cytoreduction. For the subset of intermediate-risk patients, laparoscopy for the assessment of disease resectability is reasonable.

\section{Tsonis et al. Debulking surgery in high-grade ovarian cancer}

\section{Preoperative HE4 and CA-125 level}

Both, human epididymis protein 4 (HE4) and cancer antigen $125(\mathrm{Ca}-125)$, have been suggested as markers in terms of patient selection for upfront debulking surgery in cases of high grade-serous ovarian cancer (3). High HE4 levels have been suggested as a negative prognostic factor for upfront debulking surgery and a recent study suggests an optimal cut-off of $277 \mathrm{pmol} / \mathrm{L}$ in order to evaluate highrisk of death among these women (48). Although Ca-125 remains an important marker of the presence of residual tumor load, high levels of this marker, preoperatively, suggest an accurate and sensitive circulating and tissue biomarker in cases of high-grade serous ovarian cancer and are predictive of high risk of death due to advanced ovarian cancer (10). Scientific evidence proposes that $\mathrm{Ca}-125$ regression is a valuable predictor of the efficacy of neoadjuvant chemotherapy and can identify patients who will most gain benefit by this approach $(24,48)$ (Table 1$)$.

\section{Radiographic and nuclear imaging}

Imaging techniques offer a better understanding of the extent of the disease and permit surgeons to decide whether upfront debulking surgery is optimal for patients suffering from high-grade serous ovarian cancer. More specifically, PET/CT scan is considered a necessary tool in evaluating the efficacy of cytoreduction and defining the optimal surgical timing for intervention within patients with highgrade serous ovarian cancer (24). According to scientific evidence, malignant pleural effusion and metastasis over diaphragm indicate lower chances of complete cytoreduction. Nevertheless, the evidence is not consistent and further studies need to be conducted in order to assess these imaging features' predictive value (3). Additionally, 18 fluorodeoxyglucose-positron emission tomography (18-FDG-PET) is proven to be adequate for estimating neoadjuvant chemotherapy response by quantifying the FDG uptake on tumor sites (24).

Video-assisted thoracoscopy (VATS) was suggested as optimal imaging technique in patients with pleural involvement, in order to assess the stage of the disease, whereas real-time ultrasound elastography was able to evaluate chemotherapy response but the application of the technique is quite limited $(3,24)$. Furthermore, diffusion weighted MRI (DW-MRI) offers an estimated superior predictive value for cytoreductive outcome in approximately $90 \%$ of the cases, providing essential information on serosal 
intestinal, mesenteric vascular and distant site involvement $(3,24,46)$. Last but not least, sonography has been evolved over the years, and although its use is limited, adequate pre-operative assessment of the extent of the disease can be achieved.

\section{Advantages of upfront debulking surgery}

Complete cytoreduction, meaning no macroscopically visible disease or less $10 \mathrm{~mm}$, has been well-documented for its positive predictive value in overall survival outcome $(3,24,44)$. In selected patients, upfront debulking surgery is accompanied with less morbidity compared to neoadjuvant chemotherapy with IDS $(3,49)$. Nevertheless, complete cytoreductive surgery demands higher range of surgical skills in order to achieve optimal tumor resection in abdominal and pelvic sites (44,50,51).

Specialized centers meet these criteria and offer to patients with high-grade serous ovarian cancer lower rates of morbidity as well as, higher rates of overall survival $(44,52)$.

Adequate resection of large tumor bulk results in better vascularized smaller residual tissue offering a better chemotherapeutic response, decreased chemo-resistance and enhanced host immunocompetence $(49,53)$. Thus, upfront debulking surgery offers a variety of benefits for patients with high-grade serous ovarian cancer (53).

To conclude, when spread of the disease is limited and patient profile is stable with no comorbidities, upfront debulking surgery appears to be feasible (10). In any case, surgical expertise defines the success of the procedure since experienced centers show higher rates of complete cytoreduction which offers better overall survival rates for these patients $(54,55)$.

Lower rates of chemoresistance have been welldocumented in patients who underwent upfront debulking surgery due to minimal tumor load $(3,39)$. Subsequent chemotherapy in patients with IDS found to be inadequate in patients received neoadjuvant chemotherapy followed by higher rates of recurrence demonstrating a clear advantage of primary cytoreductive surgery (56). For instance, in patients with celiac lymph node involvement, upfront debulking surgery aiming for lymphadenectomy offers higher survival rates, since positive celiac lymph nodes are accompanied with higher incidence of chemo-resistance and more advanced disease with higher rates of recurrence if not removed at the time of the surgery. In these cases, upfront debulking surgery performed by experienced surgeons offers an advantage to high-grade ovarian cancer patients over neoadjuvant chemotherapy followed by IDS (44). Current scientific data yields an impression of higher overall survivals rates in patients with advanced ovarian cancer undergoing primary cytoreductive surgery with additional intraoperative chemotherapy, regardless remaining residual disease. This evidence though is not consistent and further studies need to be conducted in order to validate this assumption (54).

\section{Advantages of IDS}

Rivals of IDS propose that neo-adjuvant chemotherapy may decrease tumor size and may offer, for this reason, improved surgical outcomes $(3,57,58)$. A recent metaanalysis proposed that IDS may succeed better results in cytoreduction rather than upfront debulking surgery $(3,59)$. Nevertheless, this evidence is not consistent and studies suggest similar overall survival rates and progression-free survival rates in both approaches $(6,60,61)$. Neoadjuvant chemotherapy though, is found to be favorable for patients with an IV disease stage with poor performance status (6). It appears that these patients are showing higher levels of quality of life (QoL), a key-point that all end-stage cancer patients are hoping for $(54,62,63)$.

On the other hand, genomic factors seem to define the success of IDS (27). Over-expression of homologous recombination DNA repair pathways, and BRCA, are associated with improved outcome of neoadjuvant chemotherapy followed by IDS $(10,64,65)$. It seems that these genomic factors ameliorate the management of patients with high-grade serous ovarian cancer offering higher overall survival rates (24). By extent, naïve to chemotherapy patients may pose a good environment in order to evaluate new regimens and biomarkers within just a few months (54).

\section{Clinical trials comparing neoadjuvant chemotherapy followed by IDS with upfront debulking surgery}

Since the first randomized phase III trial of neoadjuvant chemotherapy in advanced ovarian cancer in 2010, several studies have addressed the issue of the optimal surgical timing (66).

\section{European Organization for Research and Treatment of Cancer (EORTC) 55971}

Suggested higher cytoreduction and decreased surgical morbidity in neoadjuvant chemotherapy followed by IDS, 
while progression-free and overall survival rates showed no difference in both groups. Moreover, the same trial proposed that patients with extensive carcinomatosis or stage IV ovarian cancer were more likely to gain benefit from neoadjuvant chemotherapy followed by IDS rather than primary debulking surgery (3). Upfront debulking surgery was optimal for these patients showing higher overall survival rate in cases where no macroscopic residual disease remained at the time of surgery. In cases of neoadjuvant chemotherapy followed by IDS, no accurate surgical stage could be performed and as a result the scientific data of this RCT remain ambiguous (24).

In any case, EORTC trial revealed complete cytoreduction in $19 \%$ of the patients receiving upfront debulking surgery followed by adjuvant chemotherapy whereas $51 \%$ in patients receiving neoadjuvant chemotherapy and IDS (67).

The median overall survival and median progression-free survival measured in months were approximately 30 and 12 , respectively in both groups.

\section{CHemotherapy OR Upfront Surgery (CHORUS)}

Similar findings to EORTC 55971 were also proposed in CHORUS trial. In this trial, patients with advanced ovarian carcinoma (stage III-IV) were included and neoadjuvant chemotherapy followed by IDS found to be superior with regards to cytoreduction and surgical complications such as, postoperative death, infection, thrombosis or hemorrhage. The number of participants was 550 .

In any case, overall survival rates found to be similar in both groups (3). This study suggested that the overall survival after neoadjuvant chemotherapy was not inferior to primary surgery, and surgical morbidity and mortality were significantly reduced in this group, with a trend toward better QoL for the patients $(47,68)$. More specifically, complete cytoreduction was achieved in $17 \%$ of the patients receiving upfront debulking surgery followed by adjuvant chemotherapy whereas $43 \%$ of the patients receiving neoadjuvant chemotherapy followed by IDS $(69,70)$.

The median overall survival and median progression-free survival measured in months were approximately 23 and approximately 12 , respectively in both groups. In these first two trials, no adequate aggressive cytoreduction was able to achieved, undermining the true value of the results (24).

\section{Fapanese Clinical Oncology Group (FCOG) 0602}

Once again showed noninferiority of neoadjuvant chemotherapy followed by IDS compared to upfront debulking surgery (53). Predominantly, this trial was able to estimate lower morbidity in patients with advanced ovarian cancer undergoing neoadjuvant chemotherapy followed by IDS compared to primary debulking surgery (6). In this study the median overall survival was 49 months in upfront debulking surgery followed by adjuvant chemotherapy group while 44.3 months in the group of patients receiving IDS after neoadjuvant chemotherapy (71). The median progression-free survival was 15.1 and 16.4 months in these groups, respectively, as well as, complete cytoreduction was achieved in $12 \%$ (17 of 147 patients) in UDS group and in $64 \%$ (83 of 130 patients) in the NACT group. Regarding, optimal surgery with no residual tumor larger than $1 \mathrm{~cm}$, the trial revealed that it was achieved in 37\% (55 of 147 patients) and in $82 \%$ (92 of 147 patients) respectively in the two groups (72). More specifically, the former group of patients shoed shorter operation time, less organ resection, and less adverse events postoperatively in general (24).

\section{Surgical Complications related to Primary or Interval debulking in Ovarian Neoplasm (SCORPION)}

Moreover, the long-awaited SCORPION trial favors neoadjuvant chemotherapy followed by IDS over upfront debulking surgery (3). According to the trial, patients who underwent the latter regime, were presented with lower QoL and higher morbidity. But overall this trial suggests, that complete cytoreduction was achieved in $46 \%$ of the patients receiving upfront debulking surgery followed by adjuvant chemotherapy whereas, $58 \%$ in the neoadjuvant group $(73,74)$. Optimal cytoreduction $(<1 \mathrm{~cm}$ residual tumor) was achieved in $92,8 \%$ and $100 \%$ in both groups, respectively.

Unfortunately, data regarding overall survival and progression-free survival are still awaited.

\section{Trial of Radical Upfront Surgical Therapy in advanced ovarian cancer (TRUST trial-NCT02828618)}

Finally, TRUST trial will be able to overcome selection bias and randomize its patients.

Further scientific evidence will be published within 2024. In this trial, patients with advanced epithelial ovarian cancer, fallopian tube cancer or primary peritoneal carcinoma FIGO stage IIIB-IVB will be included providing essential data with regards to treatment options (75) (Table 2). 
Table 2 Randomized clinical trials (Phase 3) comparing upfront debulking surgery followed by adjuvant chemotherapy with interval debulking surgery (IDS) after neoadjuvant chemotherapy in cases of advanced epithelial ovarian cancer

\begin{tabular}{|c|c|c|c|c|c|c|}
\hline Study & Criteria & No of patients & OS (months & PFS (months) & $\begin{array}{c}\text { Complete } \\
\text { cytoreduction }\end{array}$ & Optimal cytoreduction \\
\hline EORTC 55971 & Stage IIIC-IV & UDS-ACT 336 & 30 both groups & 12 both groups & UDS-ACT 19\% & Not available \\
\hline \multirow{2}{*}{$\begin{array}{l}\text { The } \\
\text { CHemotherapyORUpfront } \\
\text { Surgery (CHORUS) trial }\end{array}$} & \multirow[t]{2}{*}{ 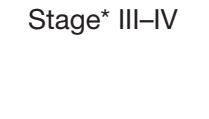 } & UDS-ACT 276 & \multirow[t]{2}{*}{23 both groups } & \multirow[t]{2}{*}{12 both groups } & UDS-ACT 17\% & \multirow[t]{2}{*}{ Not available } \\
\hline & & NACT-IDS 274 & & & NACT-IDS 43\% & \\
\hline \multirow[t]{2}{*}{ JCOG 0602 trial } & \multirow[t]{2}{*}{ Stage IIIC-IV } & UDS-ACT 149 & UDS ACT 49 & UDS ACT 15.1 & UDS-ACT 12\% & UDS-ACT 37\% \\
\hline & & NACT-IDS 152 & NACT IDS 44.3 & NACT IDS 16.4 & NACT-IDS 64\% & NACT-IDS 82\% \\
\hline \multirow[t]{2}{*}{ SCORPION trial } & \multirow{2}{*}{$\begin{array}{l}\text { Stage IIIC-IV } \\
\text { Fagotti score } 8 \\
\text { to } 12\end{array}$} & UDS-ACT 55 & \multirow[t]{2}{*}{ Not available } & \multirow[t]{2}{*}{ Not available } & UDS-ACT $46 \%$ & UDS-ACT $92.8 \%$ \\
\hline & & NACT-IDS 55 & & & NACT-IDS 58\% & NACT-IDS 100\% \\
\hline $\begin{array}{l}\text { TRUST trial, } \\
\text { NCT02828618 }\end{array}$ & Stage IIIB-IVB & 772 & \multicolumn{4}{|c|}{ Results are expected in 2024} \\
\hline
\end{tabular}

*, no histologic confirmation. UDS, upfront debulking surgery; ACT, adjuvant chemotherapy; NACT, neoadjuvant chemotherapy; IDS, interval debulking surgery; OS, median overall survival; PFS, median progression-free survival.

\section{Conclusions}

As indicated above, there is a lack of consensus regarding the optimal surgical timing in patients with high-grade serous ovarian cancer. All studies suggest personalized approach of each patients by taking into consideration a number of predictive factors that could favor one approach over the other. Understanding the prognostic factor affecting the efficacy of upfront debulking surgery will eventually offer scientific evidence with regards to patient's selection for treatment.

Algorithms should be conducted in order to estimate the efficacy of each surgical approach depending on evidence-based prognostic factors. It is imperative to assess and propose optimal surgical timing with regards to increasing overall survival rates.

Understanding prognostic factors and their influence in the progression or recurrence of the disease will facilitate specialists in terms of choosing optimal treatment option taken also into consideration QoL of the patient as a firstline aim in cases of advanced disease.

\section{Acknowledgments}

Funding: None.

\section{Footnote}

Provenance and Peer Review: This article was commissioned by the Guest Editors (Stergios Boussios and Nicholas Pavlidis) for the series "Ovarian Cancer: State of the Art and Perspectives of Clinical Research" published in Annals of Translational Medicine. The article was sent for external peer review organized by the Guest Editors and the editorial office.

Conflicts of Interest: All authors have completed the ICMJE uniform disclosure form (available at http://dx.doi. org/10.21037/atm-20-1620). The series "Ovarian Cancer: State of the Art and Perspectives of Clinical Research" was commissioned by the editorial office without any funding or sponsorship. SB serves as an unpaid editorial board member of Annals of Translational Medicine from Nov 2019 to Oct 2021. The authors have no other conflicts of interest to declare.

Ethical Statement: The authors are accountable for all aspects of the work in ensuring that questions related to the accuracy or integrity of any part of the work are appropriately investigated and resolved.

Open Access Statement: This is an Open Access article distributed in accordance with the Creative Commons Attribution-NonCommercial-NoDerivs 4.0 International License (CC BY-NC-ND 4.0), which permits the noncommercial replication and distribution of the article with 


\section{Page 8 of 11}

the strict proviso that no changes or edits are made and the original work is properly cited (including links to both the formal publication through the relevant DOI and the license). See: https://creativecommons.org/licenses/by-nc-nd/4.0/.

\section{References}

1. Boussios S, Karihtala P, Moschetta M, et al. Combined strategies with Poly (ADP-Ribose) Polymerase (PARP) inhibitors for the treatment of ovarian cancer: a literature review. Diagnostics (Basel) 2019;9:87.

2. Boussios S, Karihtala P, Moschetta M, et al. Veliparib in ovarian cancer: a new synthetically lethal therapeutic approach. Invest New Drugs 2020;38:181-93.

3. Boussios S, Karathanasi A, Cooke D, et al. PARP inhibitors in ovarian cancer: the route to "Ithaca". Diagnostics (Basel) 2019;9:55.

4. Cojocaru E, Parkinson CA, Brenton JD. Personalising Treatment for High-Grade Serous Ovarian Carcinoma. Clin Oncol (R Coll Radiol) 2018;30:515-24.

5. Singh N, McCluggage WG, Gilks CB. High-grade serous carcinoma of tubo-ovarian origin: recent developments. Histopathology 2017;71:339-56.

6. Vergote I, Coens C, Nankivell M. Neoadjuvant chemotherapy versus debulking surgery in advanced tuboovarian cancers: pooled analysis of individual patient data from the EORTC 55971 and CHORUS trials. Lancet Oncol 2018;19:1680-7.

7. Clifford C, Vitkin N, Nersesian S, et al. Multi-omics in high-grade serous ovarian cancer: Biomarkers from genome to the immunome. Am J Reprod Immunol 2018;80:e12975.

8. van Zyl B, Tang D, Bowden NA. Biomarkers of platinum resistance in ovarian cancer: What can we use to improve treatment. Endocr Relat Cancer 2018;25:R303-18.

9. Tsonis O. Endometriosis in Adolescence: Early manifestation of the Traditional Disease or a Unique Variant? Eur J Obstet Gynecol Reprod Biol 2020;247:238-43.

10. Colombo N, Sessa C, Bois A Du, et al. ESMO-ESGO consensus conference recommendations on ovarian cancer: Pathology and molecular biology, early and advanced stages, borderline tumours and recurrent disease. Int $\mathrm{J}$ Gynecol Cancer 2019;29:728-60.

11. Katchman BA, Chowell D, Wallstrom G, et al. Autoantibody biomarkers for the detection of serous ovarian cancer. Gynecol Oncol 2017;146:129-36.

12. Press JZ, De Luca A, Boyd N, et al. Ovarian carcinomas

\section{Tsonis et al. Debulking surgery in high-grade ovarian cancer}

with genetic and epigenetic BRCA1 loss have distinct molecular abnormalities. BMC Cancer 2008;8:17.

13. Naples I, Boyd N, Young S, et al. BRCA1 and BRCA2 in Ovarian Cancer: ESMO Biomarker Factsheet. OncologyproESMO.org. Available online: https:// oncologypro.esmo.org/education-library/factsheets-on biomarkers/brca1-and-brca2-in-ovarian-cancer. Accessed February 7, 2020.

14. Shi T, Wang P, Tang W, et al. Survival benefit of germline BRCA mutation is associated with residual disease in ovarian cancer. Cell Physiol Biochem 2018;47:2088-96.

15. Petrillo M, Marchetti C, De Leo R, et al. BRCA mutational status, initial disease presentation, and clinical outcome in high-grade serous advanced ovarian cancer: a multicenter study. Am J Obstet Gynecol 2017;217:334.e1-9.

16. Xu K, Yang S, Zhao Y. Prognostic significance of BRCA mutations in ovarian cancer: An updated systematic review with meta-analysis. Oncotarget 2017;8:285-302.

17. Gadducci A, Guarneri V, Peccatori FA, et al. Current strategies for the targeted treatment of high-grade serous epithelial ovarian cancer and relevance of BRCA mutational status. J Ovarian Res 2019;12:9.

18. Blok F, Dasgupta S, Dinjens WNM, et al. Retrospective study of a 16 year cohort of BRCA1 and BRCA2 carriers presenting for RRSO: Prevalence of invasive and in-situ carcinoma, with follow-up. Gynecol Oncol 2019;153:326-34.

19. Lavie O, Chetrit A, Novikov I, et al. Fifteen-year survival of invasive epithelial ovarian cancer in women with BRCA1/2 mutations - the National Israeli Study of Ovarian Cancer. Gynecol Oncol 2019;153:320-5.

20. Gungorduk K, Ozdemir A, Selcuk I, et al. Comparison of early-stage high-grade serous primary fallopian tube cancers and epithelial ovarian cancers: A multicenter study. Oncol Res Treat 2017;40:203-6.

21. Kurian AW, Hughes E, Handorf EA, et al. Breast and ovarian cancer penetrance estimates derived from germline multiple-gene sequencing results in women. JCO Precis Oncol 2017:1-12.

22. National Cancer Institute. BRCA Mutations: Cancer Risk and Genetic Testing Fact Sheet -National Cancer Institute Available online: https://www.cancer.gov/about-cancer/ causes-prevention/genetics/brca-fact-sheet. 2018. Accessed February 7, 2020.

23. Katopodis P, Chudasama D, Wander G, et al. Kinase inhibitors and ovarian cancer. Cancers (Basel) 2019;11:1357. 
24. Cho JH, Kim S, Song YS. Neoadjuvant chemotherapy in advanced ovarian cancer: Optimal patient selection and response evaluation. Chin Clin Oncol 2018;7:58.

25. Josahkian JA, Saggioro FP, Vidotto T, et al. Increased STAT1 expression in high grade serous ovarian cancer is associated with better outcome. Int J Gynecol Cancer 2018;28:459-65.

26. Jin C, Xue Y, Li Y, et al. A 2-protein signature predicting clinical outcome in high-grade serous ovarian cancer. Int J Gynecol Cancer 2018;28:51-8.

27. Zhou M, Li L, Wang X, et al. Neutrophil-to-lymphocyte ratio and platelet count predict long-term outcome of stage IIIC epithelial ovarian cancer. Cell Physiol Biochem 2018;46:178-86.

28. Chesnais M, Lecuru F, Mimouni M, et al. A pre-operative predictive score to evaluate the feasibility of complete cytoreductive surgery in patients with epithelial ovarian cancer. PLoS One 2017;12:e0187245.

29. Griffiths CT. Surgical resection of tumor bulk in theprimary treatment of ovarian carcinoma. Natl Cancer Inst Monogr 1975;42:101-4.

30. Chi DS, Eisenhauer EL, Lang J, et al. What is the optimal goal of primary cytoreductive surgery for bulky stage IIIC epithelial ovarian carcinoma (EOC)? Gynecol Oncol 2006;103:559-64.

31. Bristow RE, Del Carmen MG, Kaufman HS, et al. Radical oophorectomy with primary stapled colorectal anastomosis for resection of locally advanced epithelial ovarian cancer. J Am Coll Surg 2003;197:565-74.

32. Obermair A, Hagenauer S, Tamandl D, et al. Safety and efficacy of low anterior en bloc resection as part of cytoreductive surgery for patients with ovarian cancer. Gynecol Oncol 2001;83:115-20.

33. Peeters KC, Tollenaar RA, Marijnen CA, et al. Risk factors for anastomotic failure after total mesorectal excision of rectal cancer. Br J Surg 2005;92:211-6.

34. Yeh CY, Changchien CR, Wang JY, et al. Pelvic drainage and other risk factors for leakage after elective anterior resection in rectal cancer patients: a prospective study of 978 patients. Ann Surg 2005;241:9-13.

35. Mourton SM, Temple LK, Abu-Rustum NR, et al. Morbidity of rectosigmoid resection and primary anastomosis in patients undergoing primary cytoreductive surgery for advanced epithelial ovarian cancer. Gynecol Oncol 2005;99:608-14.

36. Kalogera E, Dowdy SC, Mariani A, et al. Multiple large bowel resections: potential risk factor for anastomotic leak. Gynecol Oncol 2013;130:213-8.
37. Seah DW, Ibrahim S, Tay KH. Hartmann procedure: is it still relevant today? ANZ J Surg 2005;75:436-40.

38. Scarabelli C, Gallo A, Franceschi S, et al. Primary cytoreductive surgery with rectosigmoid colon resection for patients with advanced epithelial ovarian carcinoma. Cancer 2000;88:389-97.

39. Takahashi O, Sato N, Miura Y, et al. Surgical indications for combined partial rectosigmoidectomy in ovarian cancer. J Obstet Gynaecol Res 2005;31:556-61.

40. Arora M, Saha S, Puthillath A, et al. Impact of radical bowel resection on survival in advanced epithelial ovarian cancer. J Clin Oncol 2005;23:5166.

41. Peiretti M, Bristow RE, Zapardiel I, et al. Rectosigmoid resection at the time of primary cytoreduction foradvanced ovarian cancer. A multi-center analysis of surgical and oncological outcomes. Gynecol Oncol 2012;126:220-3.

42. Estes JM, Leath III CA, Straughn Jr JM, et al. Bowel resection at the time of primary debulking for epithelial ovarian carcinoma: outcomes in patients treated with platinum and taxane-based chemotherapy. J Am Coll Surg 2006;203:527-32.

43. Son JH, Kong TW, Paek J, et al. Perioperative outcomes of extensive bowel resection during cytoreductive surgery in patients with advanced ovarian cancer. J Surg Oncol 2019;119:1011-5.

44. Angeles MA, Ferron G, Cabarrou B, et al. Prognostic impact of celiac lymph node involvement in patients after frontline treatment for advanced ovarian cancer. Eur J Surg Oncol 2019;45:1410-6.

45. Powless CA, Aletti GD, Bakkum-Gamez JN, et al. Risk factors for lymph node metastasis in apparent early-stage epithelial ovarian cancer: Implications for surgical staging. Gynecol Oncol 2011;122:536-40.

46. McIntosh LJ, O’Neill AC, Bhanusupriya S, et al. Prognostic significance of supradiaphragmatic lymph nodes at initial presentation in patients with stage III high-grade serous ovarian cancer. Abdom Radiol (NY) 2017;42:2513-20.

47. Makar AP, Tropé CG, Tummers P, et al. Advanced Ovarian Cancer: Primary or interval debulking? Five categories of patients in view of the results of randomized trials and tumor biology: Primary debulking surgery and interval debulking surgery for advanced ovarian cancer. Oncologist 2016;21:745-54.

48. Bairati I, Gregoire J, Plante M, et al. Performance of preoperative plasma HE4 and CA-125 levels in predicting ovarian cancer mortality in women with epithelial ovarian cancer (EOC). J Clin Oncol 2017;35:e17076. 


\section{Page 10 of 11}

49. Paulino E, Rodrigues AN, Strasser-Weippl K, et al. Barriers to primary debulking surgery for advanced ovarian cancer in Latin America. Int J Gynecol Cancer 2017;27:1645-9.

50. Biacchi D, Accarpio F, Ansaloni L, et al. Upfront debulking surgery versus interval debulking surgery for advanced tubo-ovarian high-grade serous carcinoma and diffuse peritoneal metastases treated with peritonectomy procedures plus HIPEC. J Surg Oncol 2019;120:1208-19.

51. Schneider S, Heikaus S, Harter P, et al. Serous tubal intraepithelial carcinoma associated with extraovarian metastases. Int J Gynecol Cancer 2017;27:444-51.

52. Bromley AB, Altman AD, Chu P, et al. Architectural patterns of ovarian/pelvic high-grade serous carcinoma. Int J Gynecol Pathol 2012;31:397-404.

53. Cantrell LA, Pfisterer J, Boggess J, et al. Interpreting Randomized Clinical Trials in Gynecologic Oncology Surgery: Does One Size Fit All? Am Soc Clin Oncol Educ Book 2019;39:342-50.

54. Leary A, Cowan R, Chi D, et al. Primary Surgery or Neoadjuvant Chemotherapy in Advanced Ovarian Cancer: The Debate Continues. Am Soc Clin Oncol Educ Book 2016;35:153-62.

55. Glaze S, Nation J, Köbel M. Type-specific response to neoadjuvant chemotherapy: Ovarian high-grade serous carcinoma versus colorectal mucinous carcinoma. J Obstet Gynaecol Can 2012;34:678-82.

56. Eggink FA, Koopmans CM, Nijman HW. Surgery for patients with newly diagnosed advanced ovarian cancer: Which patient, when and extent? Curr Opin Oncol 2017;29:351-8.

57. Feng $Z$, Wen $H$, Bi R, et al. Prognostic impact of the time interval from primary surgery to intravenous chemotherapy in high grade serous ovarian cancer. Gynecol Oncol 2016;141:466-70.

58. Böhm S, Faruqi A, Said I, et al. Chemotherapy response score: Development and validation of a system to quantify histopathologic response to neoadjuvant chemotherapy in tubo-ovarian high-grade serous carcinoma. J Clin Oncol 2015;33:2457-63.

59. Bogani G, Maggiore ULR, Paolini B, et al. The detrimental effect of adopting interval debulking surgery in advanced stage low-grade serous ovarian cancer. J Gynecol Oncol 2019;30:e4.

60. Zhang J, Liu N, Zhang A, et al. Potential risk factors associated with prognosis of neoadjuvant chemotherapy followed by interval debulking surgery in stage IIIc-IV high-grade serous ovarian carcinoma patients. J Obstet

\section{Tsonis et al. Debulking surgery in high-grade ovarian cancer}

Gynaecol Res 2018;44:1808-16.

61. Chung YS, Park SY, Lee JY, et al. Outcomes of non-high grade serous carcinoma after neoadjuvant chemotherapy for advanced-stage ovarian cancer: A Korean gynecologic oncology group study (OV 1708). BMC Cancer 2019;19:341.

62. Xu X, Deng F, Lv M, et al. The number of cycles of neoadjuvant chemotherapy is associated with prognosis of stage IIIc-IV high-grade serous ovarian cancer. Arch Gynecol Obstet 2017;295:451-8.

63. Mueller JJ, Kelly A, Zhou Q, et al. Intraperitoneal chemotherapy after interval debulking surgery for advanced-stage ovarian cancer: Feasibility and outcomes at a comprehensive cancer center. Gynecol Oncol 2016;143:496-503.

64. Alcarraz C, Muñiz J, Mas L, et al. Optimal cytoreduction in advanced ovarian cancer treated with dose-dense paclitaxel and carboplatin followed by interval surgery at the Peruvian national institute of neoplastic diseases. Rev Peru Med Exp Salud Publica 2018;35:46-54.

65. Petrillo M, Paris I, Vizzielli G, et al. Neoadjuvant chemotherapy followed by maintenance therapy with or without bevacizumab in unresectable high-grade serous ovarian cancer: A case-control study. Ann Surg Oncol 2015;22:S952-8.

66. Vergote I, Trope CG, Amant F, et al. Neoadjuvant chemotherapy or primary surgery in stage IIIC or IV ovarian cancer. N Engl J Med 2010;363:943-53.

67. Yoneoka Y, Ishikawa M, Uehara T, et al. Treatment strategies for patients with advanced ovarian cancer undergoing neoadjuvant chemotherapy: Interval debulking surgery or additional chemotherapy? J Gynecol Oncol 2019;30:e81.

68. Kehoe S, Hook J, Nankivell M, et al. Primary chemotherapy versus primary surgery for newly diagnosed advanced ovarian cancer (CHORUS): An open- label, randomised, controlled, non-inferiority trial. Lancet 2015;386:249-57.

69. Liu YL, Filippova OT, Zhou Q, et al. Characteristics and survival of ovarian cancer patients treated with neoadjuvant chemotherapy but not undergoing interval debulking surgery. J Gynecol Oncol 2020;31:e17.

70. Coleridge SL, Bryant A, Lyons TJ, et al. Chemotherapy versus surgery for initial treatment in advanced ovarian epithelial cancer. Cochrane Database Syst Rev 2019;2019:CD005343.

71. Karam A, Ledermann JA, Kim JW, et al. Fifth ovarian cancer consensus conference of the gynecologic 
cancer intergroup: first-line interventions. Ann Oncol 2017;28:711-7.

72. Chern JY, Curtin JP. Appropriate recommendations for surgical debulking in stage IV ovarian cancer. Curr Treat Options Oncol 2016;17:1-10.

73. Fagotti A, Ferrandina G, Vizzielli G, et al. Phase III randomised clinical trial comparing primary surgery versus neoadjuvant chemotherapy in advanced epithelial ovarian cancer with high tumour load (SCORPION trial): Final analysis of peri-operative outcome. Eur J Cancer 2016;59:22-33.

Cite this article as: Tsonis O, Gkrozou F, Vlachos K, Paschopoulos M, Mitsis MC, Zakynthinakis-Kyriakou N, Boussios S, Pappas-Gogos G. Upfront debulking surgery for high-grade serous ovarian carcinoma: current evidence. Ann Transl Med 2020;8(24):1707. doi: 10.21037/atm-20-1620
74. Onda T, Satoh T, Saito T, et al. Comparison of treatment invasiveness between upfront debulking surgery versus interval debulking surgery following neoadjuvant chemotherapy for stage III/IV ovarian, tubal, and peritoneal cancers in a phase III randomised trial: Japan Clinical Oncology Gr. Eur J Cancer 2016;64:22-31.

75. Reuss A, Du Bois A, Harter P, et al. TRUST: Trial of radical upfront surgical therapy in advanced ovarian cancer (ENGOT ov33/AGO-OVAR OP7). Int J Gynecol Cancer 2019;29:1327-31. 